\title{
Diagnostic Laparoscopy for Right Hemidiaphragm Rupture With Laparotomy Repair: A Case Report
}

\author{
James P. Bailey ${ }^{\mathrm{a}, \mathrm{c}}$, Ryan D. Edwards ${ }^{\mathrm{b}}$
}

\begin{abstract}
Post-traumatic hemidiaphragm rupture is a rare occurrence with a high mortality rate. The gold standard for diagnosis is exploratory laparotomy, which itself carries a high mortality rate in the emergent setting, as is commonly the case following blunt or penetrating abdominopelvic trauma. In this case, a 31-year-old patient with no significant medical history, who presented following an automobile accident with shoulder and gluteal pain with negative radiologic findings, was discussed. The goal of this case was to increase awareness of a possible alternative to the exploratory laparotomy for hemidiaphragm rupture in the emergent setting associated with lower mortality due to the operation. In this case, diagnostic laparoscopy was performed, and right hemidiaphragm was diagnosed. Repair was made via laparotomy with incorporation of the laparoscopic port incisions into the laparotomy incision. Repair was made using 2-0 interrupted non-absorbable sutures with both the anterior and posterior flaps of the hemidiaphragm supported with large pledgets. The patient tolerated the operation well, and has since recovered completely. This case demonstrates the possibility of utilizing minimally invasive techniques for the diagnosis of isolated diaphragm injury. Additionally, this case highlights the inadequacies of modern imaging technologies for the diagnosis of diaphragm injury, and supports the need of improvement in this technology or an alternative modality with increased sensitivity to rule out diaphragmatic injury.
\end{abstract}

Keywords: Diaphragm rupture; Diagnostic laparoscopy; Trauma

\section{Introduction}

Post-traumatic diaphragmatic rupture is a rare finding in patients who have experienced blunt or piercing thoracoabdomi-

Manuscript accepted for publication March 25, 2016

${ }^{a}$ Michigan State University College of Human Medicine, 965 Fee Rd Room A110, East Lansing, MI 48824, USA

bUpper Peninsula Health System Marquette, General Surgery/Trauma Surgery, 580 W College Ave, Marquette, MI 49855, USA

${ }^{\mathrm{c} C}$ Corresponding Author: James P. Bailey, Michigan State University College of Human Medicine, 965 Fee Rd Room A110, East Lansing, MI 48824, USA. Email: James.Bailey@alumni.nmu.edu

doi: http://dx.doi.org/10.14740/jcs298w nal trauma, with an incidence of $0.8-1.6 \%[1-3]$ and mortality of 5-30\%. Left hemidiaphragm injury is much more common than isolated right hemidiaphragmatic injury with $80-90 \%$ of ruptures occurring on the left [4]. With right hemidiaphragm rupture, damage to the liver is almost universally present $(93 \%$ of cases), as is pneumo-hemothorax (90\%), and hemoperitoneum is common [5]. Diagnosis is rarely made pre-operatively in right hemidiaphragm rupture, with $0-10 \%$ being correctly diagnosed preoperatively. The gold standard for diagnosis is exploratory laparotomy. Surgical repair is necessary even for small defects, as the diaphragm will not spontaneously repair, and the variation in thoracic to abdominal pressures favors herniation of abdominal contents and expansion of the defect. Repair is typically performed through open laparotomy using interrupted non-absorbable sutures, but cases have been reported of laparoscopic repair [6].

Urgent or emergent laparotomy is not without risks, with a 30-day mortality of $14.9 \%$ [7]. As such, an alternative diagnostic procedure to the exploratory laparotomy is needed. A study of emergent laparoscopy for acute abdominal syndromes showed high diagnostic efficacy (89\%) with $0 \%$ mortality [8], which demonstrates a promising possible method to replace emergency exploratory laparotomy for hemidiaphragm rupture.

\section{Case Report}

The patient studied was a 31-year-old male who was the restrained passenger in a sedan which was impacted on the passenger side by a pickup truck traveling at highway speeds. The patient's vehicle experienced airbag deployment, and demonstrated significant intrusion into the passenger compartment, with portions of the door impacting the patient. Upon EMS arrival to the scene, it was determined that the patient would need to be extricated using the Jaws of Life. Due to a delay in extrication equipment arriving at the scene, the patient elected to self-extricate. The patient noticed significant pain to his right buttock and shoulder, but was walking following his extrication. He refused spinal immobilization, but accepted transport to the local trauma center.

Upon presentation to the emergency department, the patient's primary complaint was pain and tenderness to his right buttock. He additionally noted some dyspnea, some pain and tenderness to his shoulder, and some small cuts to his face that he felt were concerning. On physical exam, vital signs were 


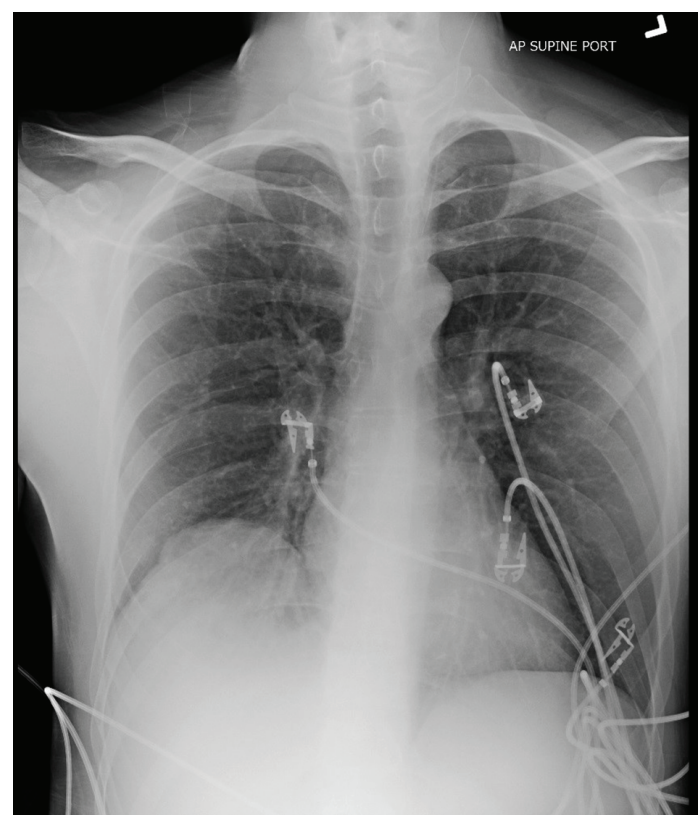

Figure 1. Pre-operative portable AP supine chest $X$-ray demonstrating elevated right hemidiaphragm.

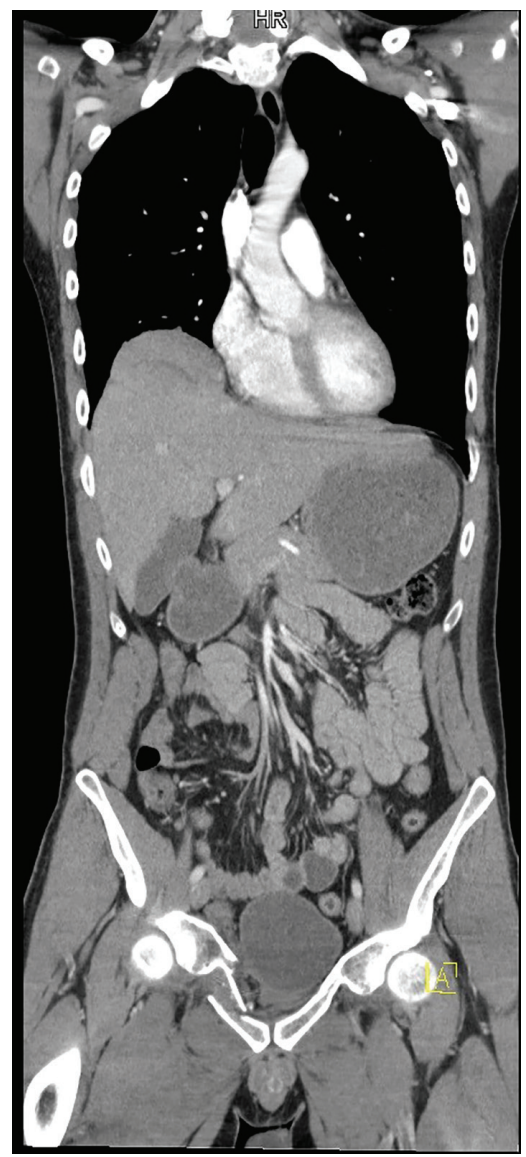

Figure 2. Coronal view of CT of chest, abdomen, and pelvis, demonstrating diaphragm asymmetry, fractured pelvic rami on right, and no hemothorax, pneumothorax, hemoperitoneum, or pneumoperitoneum.

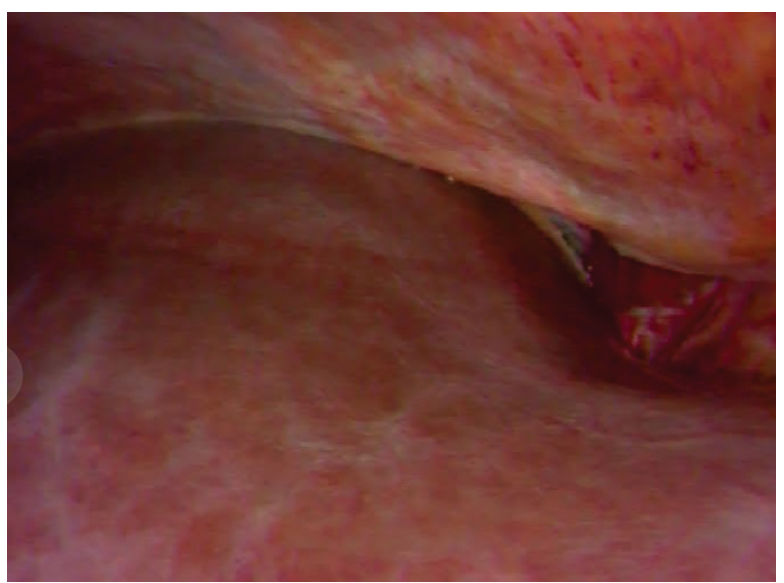

Figure 3. Laparoscopic camera image from port located just inferior to xyphoid process in midline, directed towards right upper quadrant. Liver: lower left of image. Diaphragm; upper right of image.

stable, and the patient was noted to have tenderness but no decreased strength or range of motion in both the right upper and lower extremities, some abdominal guarding without tenderness, and decreased lung sounds in the right lower lobe. A stat chest X-ray was performed (Fig. 1) which demonstrated asymmetric elevation of the right hemidiaphragm. Abdominal and pelvic X-ray demonstrated displaced fractures of the anterior rami of the pelvis, and questionable displacement of the right sacroiliac joint. These findings combined with his mechanism of injury, along with the presence of guarding and dyspnea prompted the trauma surgeon to order a CT of the head, neck, chest, abdomen, and pelvis. The CT was interpreted as a congenital asymmetrical variation of diaphragm structure (Fig. 2 ) with no acute traumatic process to the diaphragm or liver. There was no free fluid in the abdomen, no hemo-pneumothorax, and no injuries to the aorta, spine, or right shoulder.

Upon secondary survey, Murphy's sign was tested and negative, but resulted in increased tenderness to the right shoulder. Despite negative radiologic assessment, physical exam findings indicated a high likelihood of diaphragm rupture. A minimally invasive approach was decided upon for assessment of the diaphragm, and diagnostic laparoscopy was selected as the procedure of choice.

The patient was appropriately prepared for surgery, and a single $5 \mathrm{~mm}$ port was inserted just below and parallel to the costal margin in the right mid clavicular line. The peritoneum was inflated, and the camera was inserted. Initial examination demonstrated no free fluid or blood in the peritoneum, and the liver and right hemidiaphragm were located (Fig. 3). This direct visualization increased suspicion for hemidiaphragm rupture, but rupture could not be definitively confirmed, so a second $10 \mathrm{~mm}$ port was inserted. A raptor grasping device was utilized, and gentle anterior pressure was applied at the apparent union of the diaphragm and liver. This pressure resulted in clear visualization of the right pleural space, and collapse of the right lung (Fig. 4). With hemidiaphragm rupture confirmed, the ports were removed and the surgery was converted to open laparotomy with the incision following the costal margin from the right anterior axillary line to the left sternal bor- 


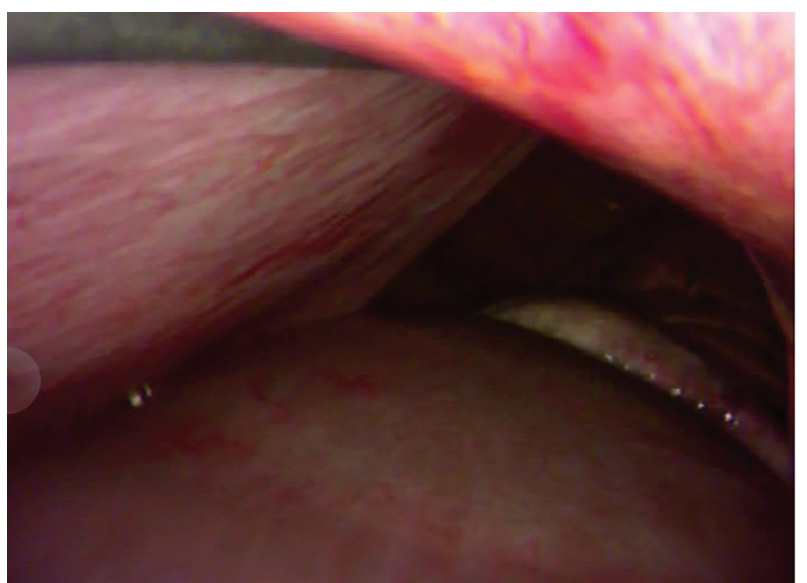

Figure 4. Laparoscopic camera image from port located just inferior to xyphoid process in midline, directed towards right upper quadrant. Liver: lower left of image. Diaphragm: upper right of image. Grasper inserted upper left. Opening demonstrates defect in diaphragm, and collapsed right lung is visible through this opening.

der, incorporating both incisions used for laparoscopy ports.

Repair was made using 2-0 interrupted non-absorbable sutures with both the anterior and posterior flaps of the hemidiaphragm supported with large pledgets. This repair allowed for increased tension to be placed on the suture without necessitating transection of the falciform ligament as is necessary in most laparotomy repairs of right hemidiaphragm rupture. There was some difficulty driving the knot to achieve adequate tension, but adequate tension was achieved with gentle posterior retraction of the liver with a malleable retractor. Following closure of the abdomen, a chest tube was inserted, and a repeat chest X-ray was obtained, which demonstrated improved symmetry of the diaphragm (Fig. 5).

The patient was admitted to the surgical floor in stable condition, with improvement in right shoulder pain. He noted that his primary complaint remained pain in his right buttock. Orthopedic surgery was consulted, and determined the pelvic fracture to be non-operative, and that the patient could weight bear. Four days after his accident, the patient was admitted to the inpatient rehabilitation unit where he remained for 2 weeks. He was discharged home from the rehab unit and has since recovered completely.

\section{Discussion}

This case demonstrates the advantages of using a minimally invasive method for diagnosis of a suspected diaphragm injury. Further assessment of this diagnostic technique should be performed, but it shows promise as an alternative to the more invasive exploratory laparotomy which remains the gold standard. With the trend of increased use of laparoscopic repair of diaphragm rupture, diagnostic laparoscopy would likely lead to shorter recovery times when compared to laparotomy, but further assessment would be necessary to confirm this.

Additionally, shortcomings of modern imaging modalities are made apparent by this case, supporting the need of a thor-

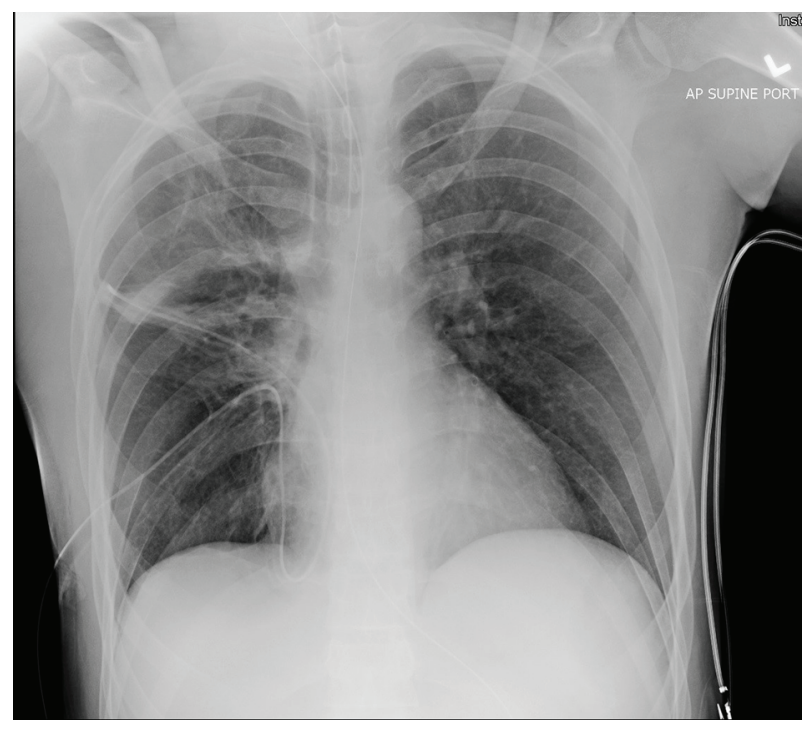

Figure 5. Post-operative portable AP supine chest $X$-ray demonstrating symmetry of diaphragm, chest tube in place in right thorax.

ough physical exam and careful history when suspecting a rare disease process.

\section{Conclusion}

This case demonstrates the need to use clinical judgement and physical exam for diagnosis when radiologic evidence does not support the diagnosis definitively. Additionally, this case demonstrates diagnostic laparoscopy as a reasonable alternative to exploratory laparotomy in the assessment of diaphragm rupture. Further assessment of this as a diagnostic method should be performed prior to any recommendation being made about its suitability to replace exploratory laparotomy for the assessment of suspected diaphragm rupture.

\section{Funding Source}

None.

\section{Conflict of Interest}

None.

\section{References}

1. Schumpelick V, Steinau G, Schluper I, Prescher A. Surgical embryology and anatomy of the diaphragm with surgical applications. Surg Clin North Am. 2000;80(1):213239, xi.

2. Rashid F, Chakrabarty MM, Singh R, Iftikhar SY. A review on delayed presentation of diaphragmatic rupture. World J Emerg Surg. 2009;4:32. 
3. Chughtai T, Ali S, Sharkey P, Lins M, Rizoli S. Update on managing diaphragmatic rupture in blunt trauma: a review of 208 consecutive cases. Can J Surg. 2009;52(3):177181.

4. Carter BN, Giuseffi J, Felson B. Traumatic diaphragmatic hernia. Am J Roentgenol Radium Ther. 1951;65(1):5672.

5. Shanmuganathan K, Killeen K, Mirvis SE, White CS. Imaging of diaphragmatic injuries. J Thorac Imaging. 2000;15(2):104-111.

6. Xenaki S, Lasithiotakis K, Andreou A, Chrysos E, Chalki- adakis G. Laparoscopic repair of posttraumatic diaphragmatic rupture. Report of three cases. Int J Surg Case Rep. 2014;5(9):601-604.

7. Saunders DI, Murray D, Pichel AC, Varley S, Peden CJ. Variations in mortality after emergency laparotomy: the first report of the UK Emergency Laparotomy Network. Br J Anaesth. 2012;109(3):368-375.

8. Al-Mulhim AS, Nasser MA, Abdullah MM, Ali AM, Kaman L. Emergency laparoscopy for acute abdominal conditions: a prospective study. J Laparoendosc Adv Surg Tech A. 2008;18(4):599-602. 